\title{
Highly Reliable Decision-Making Using Reliability Factor Feedback for Factory Condition Monitoring via WSNs
}

\author{
Zafar Iqbal $\left(\mathbb{D},{ }^{1}\right.$ Heung-No Lee ${ }^{(D)}{ }^{2}$ and Saeid Nooshabadi ${ }^{3}$ \\ ${ }^{1}$ Department of Computer Science, Michigan Technological University, Houghton, MI 49931, USA \\ ${ }^{2}$ School of Electrical Engineering and Computer Science, Gwangju Institute of Science and Technology, \\ Gwangju 61005, Republic of Korea \\ ${ }^{3}$ Department of Electrical and Computer Engineering, Michigan Technological University, Houghton, MI 49931, USA
}

Correspondence should be addressed to Heung-No Lee; heungno@gist.ac.kr

Received 22 May 2018; Revised 27 August 2018; Accepted 12 September 2018; Published 1 October 2018

Academic Editor: André de Almeida

Copyright (c) 2018 Zafar Iqbal et al. This is an open access article distributed under the Creative Commons Attribution License, which permits unrestricted use, distribution, and reproduction in any medium, provided the original work is properly cited.

\begin{abstract}
Cooperation among sensors in a wireless sensor network, deployed for industrial monitoring in an indoor scenario, is a topic of interest in the smart factory and smart city research. The indoor wireless communication channel is very harsh and the observations of all the sensors cannot be sent reliably to the base station. Failure to transmit correct sensing results to the base station may result in false alarms or missed detection of events. Therefore, we propose a cooperation scheme for the wireless sensors to send the data reliably to the base station. Our aim is to increase the reliability of the received information, reduce the probability of error, lower the overall power consumption, and keep the latency to an acceptable low level. We propose a reliability factor feedback algorithm to adjust the weight of unreliable sensors in the decision-making process. The proposed scheme is analyzed based on its latency, power consumption, and packet delivery ratio. Our results show significant improvement in the reliability of the received data, improved packet delivery, and reduced false alarm ratio for full repetition and cluster head-based cooperation. The power consumption and latency in data transmission are also kept to an acceptable low level.
\end{abstract}

\section{Introduction}

With the advancement in Internet-of-Things (IoT) and the drive towards smart factory goal, industrial wireless sensor networks (IWSNs) are becoming increasingly important in monitoring the indoor industrial area. The wireless communication link plays a very important role in transmitting the sensed information to a processing unit located in the base station (BS). A broken communication link or a fault in the sensor leads to false alarms or missed detection of events at the BS. This situation may also cause the nodes to repeat transmissions of the data or use higher transmit power leading to higher energy consumption and lower overall throughput of the network. The energy consumption per bit of the network is also affected negatively by the amount of data transmitted by the network nodes and the processing required at the receiver.

In order to improve the reliability of the received information at the BS, a number of methods have been proposed, including cooperation among wireless nodes to reduce the error due to bad channel conditions. These methods include network coding [1-3], packet loss issues in wireless traffic [4], and relay selection mechanism in networked control system (NCS) for successful cooperative transmission in industrial environments [5]. The work in [6] proposes an energy-efficient scheme to improve packet delivery by using a reliable reactive routing enhancement (R3E) protocol. For the amplify-and-forward (AF) based cooperative communication systems, an adaptive-gain M-relay AF scheme was proposed in [7] in order to achieve good error-rate performance. A solution for machine condition monitoring (MCM) in large factories, which reduces the energy consumption and improves the network throughput, was proposed in [8]. In order to reduce the probability of false alarms sent by the sensors, the work in [9] presents an IWSN-based MCM system. When cooperation among sensor nodes is used in a network consisting of multiple sensors, the aggregation and processing of data at the intermediate sensor nodes play an 
important role in the performance and energy consumption of the cooperative multihop communication system. Since all the packets are addressed to a single destination and the size of data packets is usually small, therefore, a reduction in the size of control packet overhead and the number of transmissions can improve the energy efficiency, and throughput, of the system [10-12].

Our recently proposed solution [13] reduces the probability of error in the received information at the BS by using cooperation and data aggregation at the relay nodes. However, [13] uses full repetition of the aggregated data in the cooperation group, which results in an unnecessary redundancy and leads to significant reduction in the throughput, which may be critical to the performance of the network. An improvement to [13] was proposed in [14] by performing partial repetition of the aggregated data at the intermediate nodes with the help of cluster heads. This method reduces the amount of transmissions required to transmit the same information to the BS and also reduces the latency at the expense of some reduction in performance. In the schemes in $[13,14]$, all the sensors in a cooperation group only share their observations with each other in the first phase unlike [1-3] in which the data is also received at the BS in the first phase. In the second phase, the cooperative information is sent to the BS by either using a full repetition mechanism [13] or using a selected number of cluster head $(\mathrm{CH})$ nodes [14]. In these methods, the relays only detect the received symbols and do not need to decode the symbols, unlike the method in [1-3]. The detected symbols are then used in the cooperation phase even if not correctly received. In order to ensure successful packet delivery, schemes like $[5,6]$ incur the extra overhead of retransmission but our proposed scheme does not require retransmission. Therefore, it simplifies the hardware and signal processing requirements of the relay node.

This work combines the data aggregation and cooperation mechanisms to improve the reliability of the information received at the $\mathrm{BS}$ as well as keeping the redundancy overhead to a certain limit in order to perform with low latency. In this paper, we introduce a parameter called reliability factor, which keeps track of the reliability of information received from a sensor. The reliability factor is obtained by comparing the received information from a senor with the final result, which is obtained after fusion of the information received from all the sensors within the cooperation group. Based on the reliability factor, we propose an algorithm called reliability factor feedback algorithm (RFFA) to improve the reliability of the final result by adjusting the weights of each participating sensor node in the fusion and decision-making process. A comparison of the latency in data communication and power consumption has been presented between the noncooperation, full repetition (F-Rep.), and cluster headbased cooperation schemes. Also, the packet delivery rate and false alarm rate of the proposed scheme have been compared with some previous related works.

The rest of the paper is organized as follows. Section 2 presents the system model. Section 3 describes the operation of the network. Section 4 presents the performance analysis of the proposed scheme. Section 5 presents simulation results, and Section 6 concludes the paper.

\section{System Model}

A WSN with indoor non-line-of-sight (NLOS) configuration is considered. The sensor nodes are organized into different cooperation groups based on their geographic proximity to each other. Cooperative transmission is performed within each cooperation group, $\mathscr{V}=\left\{V_{i}\right\}_{i=1}^{N}$, where $N$ is the maximum number of nodes in a cooperation group. Each node in a cooperation group is able to communicate with the BS in a dual-hop manner. The channels from a source node to an intermediate node, $\beta$, and the channels from an intermediate node to the destination, $\alpha$, are modeled as lognormal distributed Rayleigh fading channels.

2.1. Sensor Coverage and Connectivity. For the purpose of condition monitoring inside an industrial building, the sensors need to be deployed in the form of a static grid or may be deployed randomly. For the deployment of sensor network, we used the static-triangular grid deployment method [15]. The minimum number of sensors required to provide 1coverage to an area of length, $l$, and breadth, $b$, is $\mathcal{N}=$ $2 l b /\left(r^{2} \sqrt{27}\right)$, where 1-coverage means that any point in the respective area is covered by at least one sensor and $r$ represents the sensing radius of a sensor. This results in an optimal and regular deployment of sensor nodes making a triangular grid structure. In the resulting triangular grid, every three nodes with intersecting sensing ranges form an equilateral triangle with each side equal to $r \sqrt{3}$.

The minimum number of necessary and sufficient neighbor nodes of a sensor node, required to ensure the connectivity of the network, is given as $\Theta(\log \mathscr{N})$ and ranges between $0.074 \log \mathcal{N}$ and $5.1774 \log \mathcal{N}$ [16]. Accordingly, each node in a cooperation group is assumed to be able to communicate with a minimum of 6 to a maximum of 20 neighbor nodes in this paper. A node decodes the information received only from its neighbor nodes and discards the rest.

2.2. Path-Loss and Shadowing in a Factory Area. As the signal propagates through the walls, machines, and other installations inside a factory area, it creates a shadowing effect which results in the attenuation of the transmit power, referred to as path-loss, and is expressed as a ratio between the transmitted and received power. Path-loss is used to measure the received signal strength (RSS) at the receiver.

In order to find the RSS at each sensor from all other sensors in the cooperation group, we use the lognormal shadowing model. This is a generic model used to predict the propagation loss for a wide range of environments including free space and indoor factory environments [17]. The pathloss measured in $\mathrm{dB}$ at a distance $d$ from the transmitter is given by

$$
P L_{d B}(d)=P L_{d B}\left(d_{0}\right)+10 \eta \log _{10}\left(\frac{d}{d_{0}}\right)+X_{\sigma, d B},
$$

where $P L_{d B}$ is the path-loss in $d B, \eta$ is the path-loss exponent indicating the rate of decay of the mean signal with respect to distance, $d_{0}$ is a reference distance, and $X_{\sigma, d B}$ is a zeromean Gaussian random variable with standard deviation $\sigma$ 
representing the shadowing effect. In (1), $P L_{d B}\left(d_{0}\right)$ is the path-loss in $\mathrm{dB}$ at a reference distance $d_{0}$, which is calculated using the Friis free-space propagation model [18]. It is used to model the line-of-sight (LOS) path-loss incurred in the channel, given as

$$
P_{r}\left(d_{0}\right)=P_{t} \frac{G_{t} G_{r} \lambda^{2}}{\left(4 \pi d_{0}\right)^{2} L},
$$

where $P_{r}\left(d_{0}\right)$ is the received signal power in Watts, $P_{t}$ is the transmitted signal power in Watts, and $G_{t}$ and $G_{r}$ are the gains of transmitter and receiver, respectively. $\lambda$ is the wavelength of the carrier in meters, and $L$ is the system losses which are not associated with propagation loss. Generally, it is more convenient to work in log domain because the transmitted and received powers are usually available in $\mathrm{dBm}$ and the antenna gains in $\mathrm{dBi}$. Therefore, the Friis free-space equation is given in log domain as

$$
\begin{aligned}
P L_{d B}\left(d_{0}\right)= & P_{t, d B}+10 \log _{10}\left(G_{t}\right)+10 \log _{10}\left(G_{r}\right) \\
& +20 \log _{10}(\lambda)-20 \log _{10}\left(4 \pi d_{0}\right) \\
& -10 \log _{10}(L) .
\end{aligned}
$$

In (3), $G_{t}, G_{r}$, and $L$ are taken equal to 1 as we consider unit gain antennas and the internal system losses are considered as 1 , whereas the reference distance $d_{0}$ is taken as $1 \mathrm{~m}$. Using (3) in (1) and the parameters suitable for indoor factory nonline-of-sight (NLOS) environments, we can compute the RSS at a receiving node as

$$
\begin{aligned}
P L_{d B}(d)= & P_{t, d B}+10 \log _{10}\left(G_{t}\right)+10 \log _{10}\left(G_{r}\right) \\
& +20 \log _{10}(\lambda)-20 \log _{10}\left(4 \pi d_{0}\right) \\
& -10 \log _{10}(L)+10 \eta \log _{10}\left(\frac{d}{d_{0}}\right)+X_{\sigma, d B}
\end{aligned}
$$

\section{Network Operation}

The operation of the network is controlled by using the organize and operate protocol (OOP) [13] and also OOP with cluster heads (OOP-CH) [14]. In these protocols, the nodes are first organized into cooperation groups. Then in OOP$\mathrm{CH}$, the BS chooses a number of cluster heads based on the received signal strength information (RSSI). After this, the normal operation of sensing and sending the data to the BS by using a two-phase cooperation mechanism starts. In the case of OOP, the sensing and transmit operations start after the nodes are organized into cooperation groups as there are no $\mathrm{CH}$ nodes used. A sequence flow diagram of the OOP$\mathrm{CH}$ protocol is presented in Figure 1. The OOP has a similar flow except the controls necessary for $\mathrm{CH}$-based cooperation. Upon receiving the cooperative packets from all the intermediate nodes, the BS performs majority voting-based fusion and makes a decision based on the received information.

3.1. Phase 1. In this phase, a sensor first senses the surrounding area for the intended information. After this, every sensor in the cooperation group shares its sensed information with the intermediate nodes ( $\mathscr{C}$ nodes in the case of $\mathrm{CH}$ based cooperation, as shown in Figure 2) present in its neighborhood by referring to its neighbor list, using BPSK modulation and TDMA scheme. The received signal $r_{i, j}$ at node $V_{j}$, from node $V_{i}$, in phase 1 is

$$
r_{i, j}=\sqrt{E_{s 1}} v_{i} \beta_{i, j}+n_{i, j}
$$

where $E_{s 1}$ is the transmitted symbol energy in phase $1, v_{i}$ is the binary information sent from node $V_{i}$, and $n_{i, j}$ is the AWGN with power spectral density, $N_{0}$. The data packet in this phase contains the floor number, sensor ID, time-of-origin (TOO), and the sensed alarm information. See [13] for detail of the data packet.

3.2. Phase 2. In this phase, each intermediate node $(\mathscr{C}$ node in the case of $\mathrm{CH}$-based cooperation, as shown in Figure 2) $V_{j}$ makes a cooperative data packet by combining the information received from the cooperating nodes within its cooperation group, $\mathscr{V}$, during the first phase. Then the cooperative data packet denoted by $x_{j}$ at a node $j$, which is formed by the aggregation of the received and amplified packets, is transmitted to the BS in a TDMA manner. The received signal at the $\mathrm{BS}, y_{j, D}$, can be written as

$$
y_{j, D}=\sqrt{E_{s 2}} x_{j} \alpha_{j, D}+n_{j, D}
$$

where $\alpha_{j, D}$ represents the lognormal fading channel coefficient from node $V_{j}$ to the BS and $E_{s 2}$ is the transmitted symbol energy in phase 2. $n_{j, D}$ is the AWGN at destination $D$ from node $j$, with power spectral density, $N_{0}$. The signal from the source node $i$, relayed via the relay node $j$ and received at the destination $D$, can be written as

$$
y_{j, D}=\sqrt{E_{s 1} E_{s 2}} \zeta_{i, j} \alpha_{j, D} \beta_{i, j} v_{i}+n_{j, D}^{\prime}
$$

where $\zeta_{i, j}=1 / \sqrt{E_{s 1}\left|\beta_{i, j}\right|^{2}+N_{0, i, j}}$ represents the amplification factor used at relay node $V_{j}$ with a corresponding source node $V_{i}$ and $n_{j, D}^{\prime}=\left(\sqrt{E_{s 2}} / \sqrt{E_{s 1}\left|\beta_{i, j}\right|^{2}+N_{0, i, j}}\right) \alpha_{j, D} n_{i, j}+$ $n_{j, D}$. Since the noise terms $n_{i, j}$ and $n_{j, D}$ can be assumed independent, then the equivalent noise $n_{j, D}^{\prime}$ is a zero-mean complex Gaussian random variable with variance given as $N_{0}^{\prime}=\left(\left(\left(E_{s 2}\left|\alpha_{j, D}\right|^{2}\right) /\left(E_{s 1}\left|\beta_{i, j}\right|^{2}+N_{0, i, j}\right)\right)+1\right) N_{0}$.

3.3. Reliability Factor Feedback Algorithm. The base station receives the information from the intermediate nodes (either $\mathscr{C} \mathrm{CH}$ nodes or all $N$ node in the cooperation group), decodes the information, and combines it at the fusion center by using majority rule decision. In the case of OOP- $\mathrm{CH}$, a majority rule decision, which consists of votes from $\mathscr{C} \mathrm{CH}$ nodes in the cooperation group $\mathscr{V}$, is mathematically represented as

$$
R(i)=\arg \max _{X} \sum_{j=1}^{\mathscr{C}} w_{j} I\left(y_{j}(i)=X\right)
$$




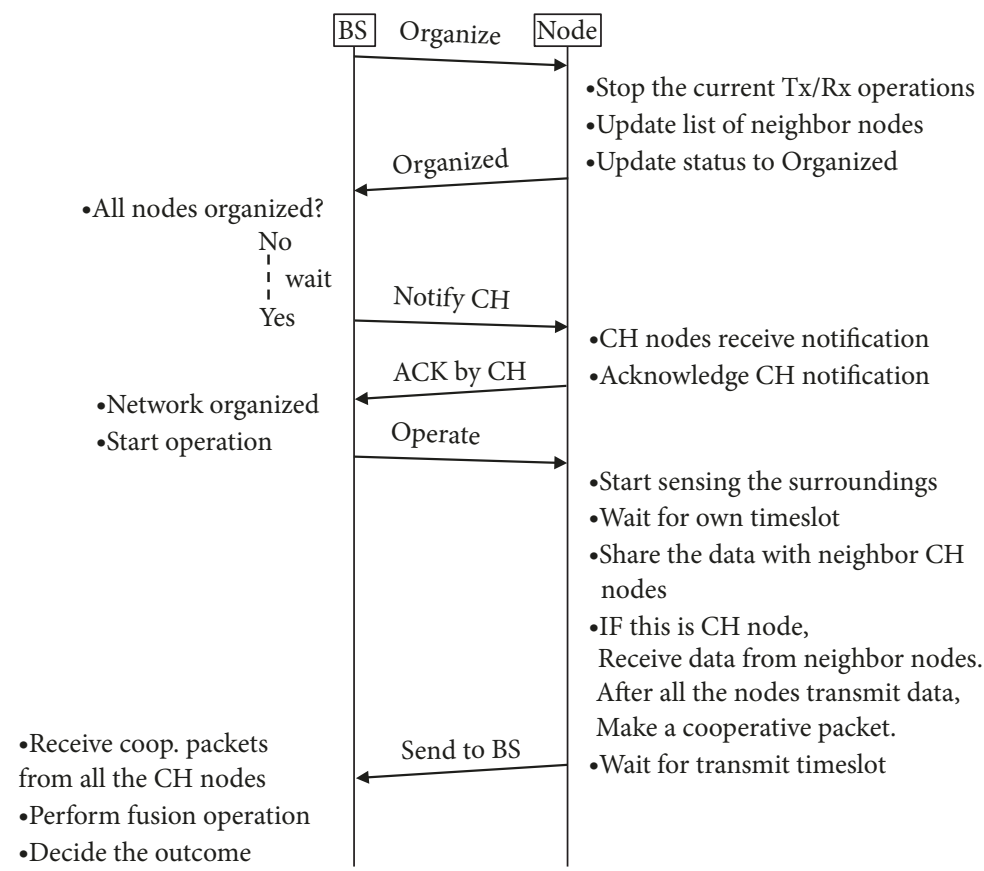

FIGURE 1: The proposed organize and operate protocol with cluster heads (OOP-CH) for WSN.

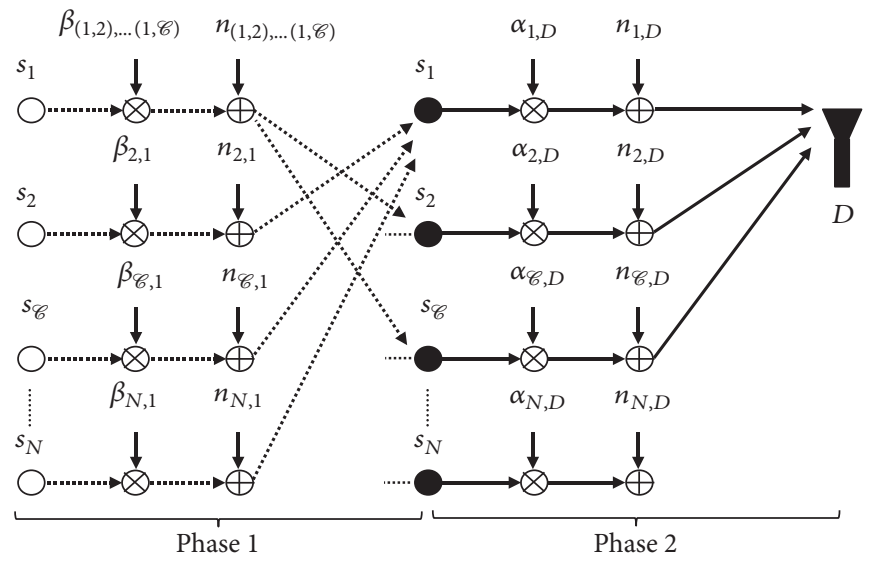

Figure 2: Depiction of the two-phase cooperative communication system. In Phase 1, for example, the sensor $s_{1}$ sends its information to all $\mathrm{CH}$ sensors $\left(s_{2}, s_{3}, \ldots, s_{\mathscr{C}}\right)$ during its allocated time slot. Similarly, all the other sensors transmit their information to $\mathrm{CH}$ sensors. In Phase 2 , only the $\mathrm{CH}$ sensors then make a cooperative packet of the aggregated data and transmit it to the destination, $D$.

where $y_{j}(i)$ is the $i$ th cooperative symbol received from a sensor $j, w_{j}$ is the weight associated with the reliability of information received from each intermediate node, and $I($. is an indicator function. In the case of OOP, the summation in (8) is taken over all $N$ nodes in the cooperation group.

In order to achieve a highly reliable result after fusion of the received information, we propose a reliability factor feedback algorithm. In this algorithm we compute a reliability factor for each of the intermediate nodes $(\mathscr{C}$ nodes in the case of $\mathrm{CH}$-based cooperation and $N$ nodes in the case of F-Rep. cooperation) by using the result obtained after information fusion at the fusion center. The reliability factor is then fed back to the majority rule fusion and used as the weight $w_{j}$ of each intermediate node involved in the fusion process. Figure 3 shows a block diagram of the proposed fusion mechanism with the RFFA for OOP-CH scheme. In case of OOP, the number of sensors are $N$ instead of $\mathscr{C}$.

3.3.1. Computing the Reliability Factor. The following steps are taken in order to compute the reliability factor, $\gamma$ for each sensor.

Definition 1. An error report, $\varepsilon$, is defined as a reported observation by a sensor which is different from the final decision after majority rule fusion.

(1) Find all the error reports made by each sensor in one transmission. 


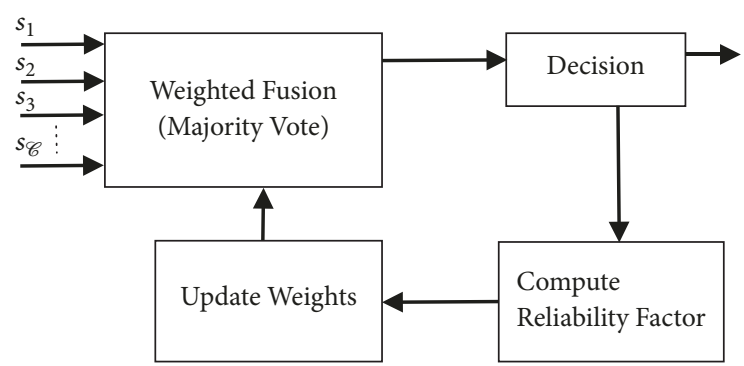

FIGURE 3: Block diagram of the reliability factor feedback algorithm.

(2) Divide the number of error reports of each sensor by the total number of sensors in a cooperation group.

(3) Subtract the computed value from 1 to get the reliability factor.

$$
\gamma_{i, j}=1-\left(\frac{\varepsilon_{i, j}}{N}\right)
$$

3.3.2. Updating the Weights. After the reliability factor has been computed, it is used as the weight of each sensor in the decision-making process. A high reliability factor value of a sensor results in heavier weight of the corresponding sensor in the voting and decision-making process.

(1) Take an average of the reliability factors of each sensor in the cooperation group, received from the intermediate sensors involved in the fusion process. Divide the computed value by the total number of intermediate sensors ( $\mathscr{C}$ nodes in the case of $\mathrm{CH}$ based cooperation and $N$ nodes in the case of F-Rep. cooperation).

$$
\begin{aligned}
w_{j(\text { F-Rep. })} & =\frac{\sum_{i=1}^{N} \gamma_{i, j}}{N N} \\
w_{j(c h)} & =\frac{\sum_{i=1}^{N} \gamma_{i, j}}{N \mathscr{C}}
\end{aligned}
$$

(2) Feedback the computed weights $w_{j}$ to the fusion process given by (8) and as shown in Figure 3.

\section{Performance Analysis}

In this section, we analyze the performance of the proposed $\mathrm{CH}$ cooperation scheme with the help of symbol error rate (SER), latency in transmission, and power consumption.

4.1. Symbol Error Rate. The proposed system is a dual-hop communication system with multiple branches. Each relay has multiple branch inputs and repeats the symbols for its neighbor nodes, in a single branch output by using AF scheme, in a TDMA manner to ensure orthogonality of the transmission. The resulting SER can be approximated by the following equation derived in our previous work [13, Theorem 1],

$$
\begin{aligned}
& P_{s}\left(\gamma_{e q, j, D}\right) \\
& \quad=F\left(1+\frac{g_{P S K}}{N_{0}^{\prime} \sin ^{2} \theta}\left(\frac{\sigma_{j, D}^{2} \prod_{i=1}^{N-1} \sigma_{i, j}^{2}}{\prod_{i=1}^{N-1} \sigma_{i, j}^{2}+\sigma_{j, D}^{2}+1}\right)\right)
\end{aligned}
$$

where $F(x(\theta))=(1 / \pi) \int_{0}^{(M-1) \pi / M}(1 / x(\theta)) d \theta, M$ is the modulation symbol size, $g_{P S K}=\sin ^{2}(\pi / M), \gamma_{e q, j, D}$ represents the instantaneous SNR per relay node at the destination, and $\sigma_{i, j}^{2}, \sigma_{j, D}^{2}$ are the variances of the Rayleigh fading channel coefficients $\beta_{i, j}$ and $\alpha_{j, D}$, respectively. For the proof of the result in (11), please see [13] Appendix A.

From (11), we get $P_{s}$ as the probability of error in the information received from an intermediate sensor. Since this is a cooperative system with multiple nodes sending information about the same event, therefore, the number of votes needed to decide the final outcome, i.e., the majority, is $l=\lceil(N+1) / 2\rceil$ in the case of F-Rep. cooperation and $l=\lceil(\mathscr{C}+$ $1) / 2\rceil$ in the case of $\mathrm{CH}$-based cooperation. The respective probability of error in the consensus can be computed by using the Binomial theorem,

$$
\begin{aligned}
P_{\text {eF-Rep. }}(N) & =\sum_{m=l}^{N}\left(\begin{array}{l}
N \\
m
\end{array}\right) P_{s}^{m}\left(1-P_{s}\right)^{N-m} \\
P_{e C H}(\mathscr{C}) & =\sum_{m=l}^{\mathscr{C}}\left(\begin{array}{l}
\mathscr{C} \\
m
\end{array}\right) P_{s}^{m}\left(1-P_{s}\right)^{\mathscr{C}-m}
\end{aligned}
$$

4.2. Latency. For the sake of a fair comparison between noncooperative and cooperative systems, we assume a traditional relay-based scheme with dual-hop communication for the noncooperative method. In this scheme, a relay node forwards the data from a source node in the second hop towards the BS without any cooperative mechanism involved. Let $B$ represent the number of bits per symbol, and the symbol duration is given by $T_{s}=1 / f_{s}$, where $f_{s}$ is the symbol rate. Then, the throughput in case of noncooperation $\left(T_{n c}\right)$, F-Rep. cooperation $\left(T_{F-R e p}\right)$, and CH-based cooperation $\left(T_{c h}\right)$ dualhop communication can be written as

$$
\begin{aligned}
T_{n c} & =\frac{N B}{N T_{s}+N T_{s}} \mathrm{bps} \\
T_{\text {F-Rep. }} & =\frac{N B}{N T_{s}+N N T_{s}} \mathrm{bps} \\
T_{c h} & =\frac{N B}{N T_{s}+\mathscr{C N T}} \mathrm{bps}
\end{aligned}
$$

where the time taken by two hops to transmit the symbol to BS is represented by the addition in the denominator. Since in F-Rep. cooperation and $\mathrm{CH}$ cooperation, each intermediate node relays the data of $N$ or $\mathscr{C}$ nodes in the second phase, respectively, it results in the additional $N$ or $\mathscr{C}$ in the denominator for $T_{F-R e p .}$ and $T_{c h}$. The delay incurred in transmitting $N$ packets to the BS in the case of noncooperation $\left(\mathscr{D}_{n c}\right)$ F-Rep. 
cooperation $\left(\mathscr{D}_{\text {F-Rep. }}\right)$ and $\mathrm{CH}$ cooperation $\left(\mathscr{D}_{c h}\right)$ schemes can then be computed as

$$
\begin{aligned}
\mathscr{D}_{n c} & =\frac{N \times \text { size of data packet (bits) }}{T_{n c} \text { (bps) }} \\
\mathscr{D}_{\text {F-Rep. }} & =\frac{N \times \text { size of data packet (bits) }}{T_{F-\text { Rep. }} \text { (bps) }} \\
\mathscr{D}_{c h} & =\frac{N \times \text { size of data packet (bits) }}{T_{c h} \text { (bps) }}
\end{aligned}
$$

4.3. Power Consumption. In this subsection, we compute the power consumption of the proposed system. In order to simplify our analysis, we do not take into account the power consumed by each sensor during sensing, and the power consumed by the usual processing operations at the BS and the intermediate nodes as these power consumption operations are common among all the schemes compared here in this paper. Hence, we will compute the power consumed in transmitting the information to the BS and the information fusion operation at the BS and compare the noncooperation, F-Rep. cooperation, and $\mathrm{CH}$ cooperation schemes. We first compute the energy consumed by these operations and then convert it to power in $\mathrm{dBm}$ units as it is easy to visualize. Let $E_{t}, E_{i}$, and $E_{r}$ represent the energy consumed by the transmit operation by a sensor, idle listening, and reception at a sensor node/BS, respectively. In the case of noncooperative dual-hop communication, each node transmits with energy $E_{t}$ in phase 1 and the other $N-1$ nodes receive this information with energy $E_{r}$. In phase 2, each relay node transmits with energy $E_{t}$ to the BS while the other $N-1$ nodes remain idle, and the BS receives each node's data with energy $E_{r}$. Thus the total power consumed $\left(\mathscr{P}_{n c}\right)$ is given as

$$
\begin{aligned}
& \mathscr{P}_{n c}=10 \log _{10}\left(\frac{1000}{N T_{s} \times 1 \mathrm{~W}} \times\left(N\left(E_{t}+(N-1) E_{r}\right)\right.\right. \\
& \left.\left.\quad+N\left(E_{t}+(N-1) E_{i}+E_{r}\right)\right)\right) .
\end{aligned}
$$

For computing the power consumption of the cooperative dual-hop communication, let $E_{f}$ represent the energy consumed by the fusion operation at the BS. The total power consumed by F-Rep. cooperation ( $\mathscr{P}_{\text {F-Rep. }}$ ) and $\mathrm{CH}$ cooperation $\left(\mathscr{P}_{c h}\right)$ is given as

$$
\begin{aligned}
& \mathscr{P}_{\text {F-Rep. }}=10 \log _{10}\left(\frac{1000}{N T_{s} \times 1 \mathrm{~W}}\right. \\
& \times\left(N\left(E_{t}+(N-1) E_{r}\right)\right. \\
& \left.\left.\quad+N\left(E_{t}+(N-1) E_{i}+E_{r}\right)+N N E_{f}\right)\right) \\
& \mathscr{P}_{c h}=10 \log _{10}\left(\frac{1000}{N T_{s} \times 1 \mathrm{~W}} \times\left(N\left(E_{t}+(N-1) E_{r}\right)\right.\right. \\
& \left.\left.\quad+\mathscr{C}\left(E_{t}+(N-1) E_{i}+E_{r}\right)+\mathscr{C} N E_{f}\right)\right)
\end{aligned}
$$

TABLE 1: Simulation parameters.

\begin{tabular}{lc}
\hline Parameter & Value \\
\hline Total area & $100 \mathrm{~m} \times 100 \mathrm{~m}$ \\
No. of cooperation nodes, $N$ & 12,18 \\
No. of cluster head nodes, $\mathscr{C}$ & 3,5 \\
Carrier frequency & $2.4 \mathrm{GHz}$ (ISM Band) \\
Transmit power, $E_{s 1}, E_{s 2}$ & $1 \mathrm{~mW}$ \\
Standard deviation, $\sigma$ & 7 (Indoor NLOS) \\
Path-loss exponent, $\eta$ & 3 (Indoor NLOS) \\
Sensing radius of each sensor, $r$ & $18 \mathrm{~m}$ \\
\hline
\end{tabular}

where $N N$ and $\mathscr{C} N$ terms in the numerator of (17) and (18) represent the number of multiply-and-accumulate operations performed to compute the fusion result for either $N$ or $\mathscr{C}$ cooperative packets each containing $N$ number of observations as given in (8). Also, in the second term in the numerator of (18), $N$ is replaced by $\mathscr{C}$ as there are $\mathscr{C} \mathrm{CH}$ nodes transmitting to the BS instead of all the $N$ relay nodes.

\section{Simulation Results}

In order to carry out simulations, we assumed an indoor communication environment with an area of $100 \mathrm{~m} \times 100 \mathrm{~m}$. The indoor area is assumed to contain heavy machines and hard partitioned walls. Rayleigh fading with NLOS lognormal shadowing channel parameters (standard deviation $\sigma=7$, path-loss exponent $\eta=3$ ) is used to model the indoor factory environment [19]. The ISM band carrier frequency of $2.4 \mathrm{GHz}$ is used with a transmit power of $1 \mathrm{~mW}$. Suppose that a fault in the operation or state of the machine at a certain location is evident from higher temperature at that location. We use Gaussian random fields to model this information over the entire area. As the field varies from high temperature to low, four different kinds of alarms, i.e., Danger, Warning, Caution, and $\mathrm{OK}$, are generated, respectively. For the simulation, we choose a cooperation group of either 18 or 12 nodes with 5 or $3 \mathrm{CH}$ nodes, respectively, and the results are averaged over 10,000 sensing operations. To observe the advantage of using RFFA clearly, we deliberately introduced error in the transmission from 3 of the 18 nodes for F-Rep. cooperation and 1 of the $5 \mathrm{CH}$ nodes for $\mathrm{CH}$ cooperation in the second phase. The simulation parameters are summarized in Table 1.

5.1. SER. In order to verify our approximated numerical result in (13), we simulate a cooperation group of 18 nodes with $3 \mathrm{CH}$ nodes. Figure 4 shows the plot of the result obtained in (13) compared with the SER obtained from simulation of the $\mathrm{CH}$ cooperation scenarios. A similar result for F-Rep. cooperation (12) was published in our previous work [13] and is not shown here. The result shows that the approximation works well to predict the performance of the proposed scheme.

5.2. Latency and Power Consumption. In order to compute the latency and power consumption of the proposed scheme in a practical scenario, we take the example of a Zigbee 


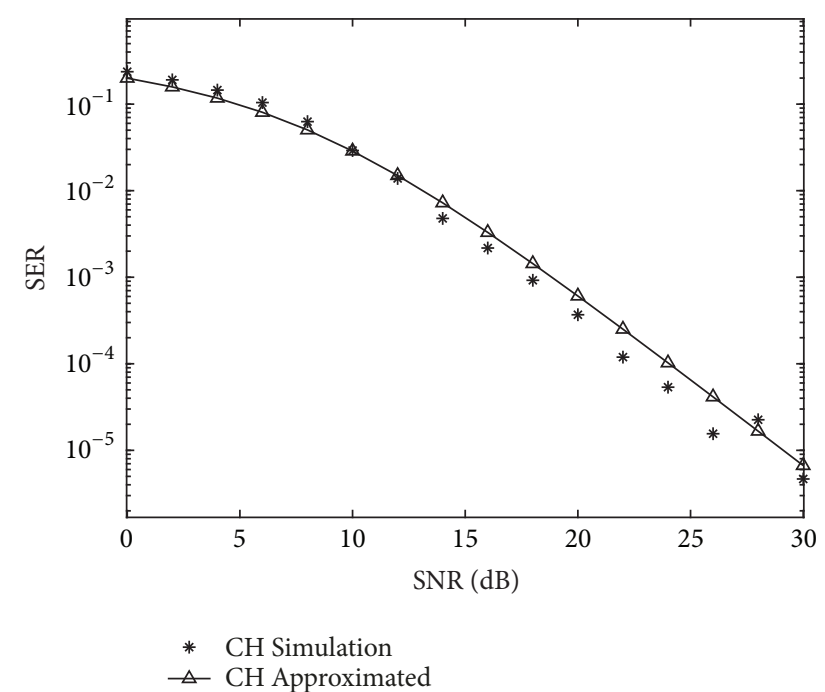

FIGURE 4: Comparison of $\mathrm{CH}$ simulation and the approximated result, given in (13). In this experiment, $3 \mathrm{CH}$ nodes were chosen from a cooperation group of 18 .

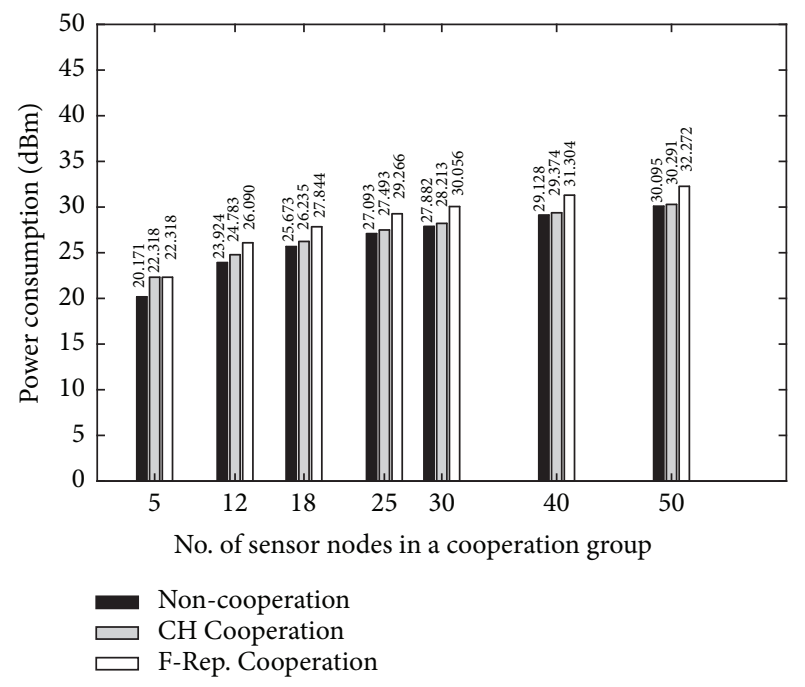

FIgURE 5: Comparison of power consumption results of the proposed $\mathrm{CH}$ scheme, F-Rep. scheme, and no cooperation scheme.

(IEEE 802.15.4) based implementation [20]. For the sensor nodes, we utilize the data from Silicon Lab's EFR32 Mighty Gecko Mesh Networking Wireless SoC, which can be used to implement a Zigbee, Bluetooth, Thread, or a proprietary $2.4 \mathrm{GHz}$ wireless sensor network [21]. Therefore, we take $T_{s}$ $=50 \mu \mathrm{s}[20], E_{t}=0.05 \mu \mathrm{J}, E_{i}=0.14 \mathrm{~nJ}, E_{r}=1.02 \mu \mathrm{J}[21]$, and $E_{f}=0.665 \mu \mathrm{J}$ [22]. The latency and power consumption results of the F-Rep. cooperation (each node transmits a cooperative packet in phase 2 to the BS, as in [13]), $\mathrm{CH}$ cooperation (each $\mathrm{CH}$ node transmits a cooperative packet in phase 2 to the BS, as in [14]), and the relayed transmission (a relay node forwards the data for a source node without any cooperation mechanism) are shown in Figures 5 and 6, respectively. The results show increased latency and power

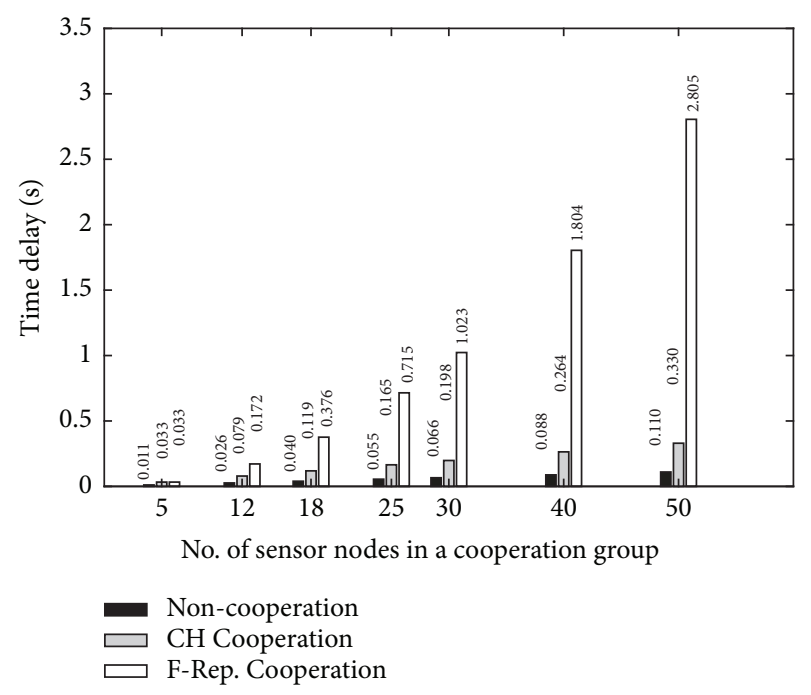

FIGURE 6: Comparison of latency results of the proposed $\mathrm{CH}$ scheme, F-Rep. scheme, and no cooperation scheme.

consumption in the case of F-Rep. cooperation and $\mathrm{CH}$ cooperation. However, the increase in power consumption in case of F-Rep. cooperation, reported in [13], has been reduced from $\sim 2 \mathrm{~dB}$ to $\sim 0.8 \mathrm{~dB}$ for $N=12$ and from $\sim 2.2 \mathrm{~dB}$ to $\sim 0.2 \mathrm{~dB}$ for $N=50$. The reduction is because the number of relay nodes in the second phase has been reduced from $N$ to $\mathscr{C}$ and only $\mathscr{C} \mathrm{CH}$ nodes now transmit to the BS in the second phase instead of all the $N$ nodes. The latency, shown in Figure 6, has been reduced from $\sim 145 \mathrm{~ms}$ to $\sim 53 \mathrm{~ms}$ for $N$ $=12$ and from $\sim 2.7 \mathrm{~s}$ to $\sim 220 \mathrm{~ms}$ for $N=50$, by using $\mathrm{CH}$ cooperation method. This is also because of the reduction in the number of relay nodes in the second phase from $N$ to $\mathscr{C}$. As the number of relay nodes reduce to a suitable number in the form of $\mathrm{CH}$ nodes, necessary to obtain cooperation benefit, it helps reduce the power consumption as well the time required to transmit all the information to the BS in order for the BS to be able to make a decision. The results of $\mathrm{CH}$ cooperation show a significant improvement in the latency and energy consumption on that reported in [13] and this will be helpful in achieving the low-latency design goal of future communication systems.

5.3. False Alarm and Packet Delivery Rates. We have used the false alarm rate (FAR) and packet delivery rate (PDR) metrics to compare our results with some of the previous works including our own work in [13]. The FAR and PDR were calculated and averaged over a range of $S N R(0$ to $30 \mathrm{~dB})$ with a total of 10,000 packets for $N=12$ (F-Rep. cooperation) and $\mathscr{C}=5$ (CH cooperation). In order to keep the comparison fair, we use the PDR result of [5], when no relay selection mechanism is used, and the PDR result for IWSN given by [6]. As shown in Table 2, the $\mathrm{CH}$ cooperation scheme shows significant improvement in the FAR when compared to [9], performs better than [8] and shows increased FAR from that reported in [13]. This work shows an increased FAR than that of [13] because the benefit of cooperation has been reduced from full repetition ( $N$ nodes) to partial repetition ( $\mathscr{C}$ nodes) 
TABLE 2: Comparison with related works.

\begin{tabular}{lccccccc}
\hline Performance Metrics & {$[4]$} & {$[5]$} & {$[6]$} & {$[8]$} & {$[9]$} & {$[13]$ F-Rep. } & {$[\mathbf{1 4}]$ CH } \\
\hline FAR & - & - & - & $3.8 \%$ & $10.5 \%$ & $1.8 \%$ & $3.1 \%$ \\
PDR & $\sim 84 \%$ & $\sim 73 \%$ & $\sim 70 \%$ & - & - & $\sim 86 \%$ & $\sim 80 \%$ \\
\hline
\end{tabular}

TABLE 3: Effect of RFFA on performance.

\begin{tabular}{lcccc}
\hline Performance Metrics & F-Rep. & WF-Rep. & CH & WCH \\
\hline FAR & $3.1 \%$ & $2.9 \%$ & $7.1 \%$ & $5.3 \%$ \\
PDR & $\sim 78 \%$ & $\sim 81 \%$ & $\sim 70 \%$ & $\sim 73 \%$ \\
\hline
\end{tabular}

in the second phase. The PDR of the $\mathrm{CH}$ cooperation scheme is higher than that reported in $[5,6]$ but lower than the PDR reported in $[4,13]$. The reason for this is [4-6] use mechanisms of retransmission, guide-path discovery, and relay selection, respectively, which increases the overhead significantly. In contrast, our work does not involve these overheads and therefore, our results show a higher PDR and lower FAR as compared to these works. Again, the PDR is lower than that of [13] because the benefit of cooperation has been reduced from full repetition ( $N$ nodes) to partial repetition ( $\mathscr{C}$ nodes) in the second phase.

Table 3 shows the FAR and PDR results of our proposed RFFA scheme in both F-Rep. $(N=18)$ and $\mathrm{CH}(\mathscr{C}=5)$ cooperation methods. Notice that the FAR and PDR of both FRep. and $\mathrm{CH}$ cooperation schemes drop down significantly from that in Table 2 because of the deliberately induced error in the cooperating nodes ( 3 nodes in case of F-Rep. and 1 node in case of $\mathrm{CH}$ cooperation). Using RFFA mitigates this problem by using the reliability factor associated with each node and improves the FAR and PDR as shown by WF-Rep. and WCH columns in Table 3. This result shows that our proposed RFFA helps in increasing the reliability of the final decision even in the presence of adversely affected sensor nodes because of communication link failure or node failure. The reason for improved FAR and PDR in both FRep. and $\mathrm{CH}$ cooperation methods is because the proposed algorithm helps reduce errors in the final decision at the BS by disregarding the information from the compromised sensors in the cooperation group.

5.4. Packet Error Rate. Figure 7 compares the packet error rate (PER) of the proposed $\mathrm{CH}$, F-Rep., relayed, and direct transmission schemes. The results show that, using the proposed RFFA, the error induced in either the intermediate nodes ( $\mathrm{CH}$ cooperation) or any of the cooperating nodes ( $\mathrm{F}$ Rep. cooperation) is successfully mitigated, as shown by the dashed lines for both WCH and WF-Rep. cooperation. The F-Rep. cooperation and $\mathrm{CH}$ cooperation schemes achieve, on average, $10^{-2}$ probability of error at almost $20 \mathrm{~dB}$ and 12 $\mathrm{dB}$ lower SNR compared with the direct (noncooperation) schemes, respectively. Despite the extra energy $(\sim 0.5 \mathrm{~dB}$ for $\mathrm{CH}$ and $\sim 2 \mathrm{~dB}$ for F-Rep., 18 nodes, Figure 5) spent by the network in performing cooperation, the amount of energy saving that can be achieved by using the $\mathrm{CH}$ cooperation and F-Rep. cooperation is $\sim 11 \mathrm{~dB}$ and $\sim 18 \mathrm{~dB}$, respectively. The reduced energy saving in $\mathrm{CH}$ scheme is a result of the

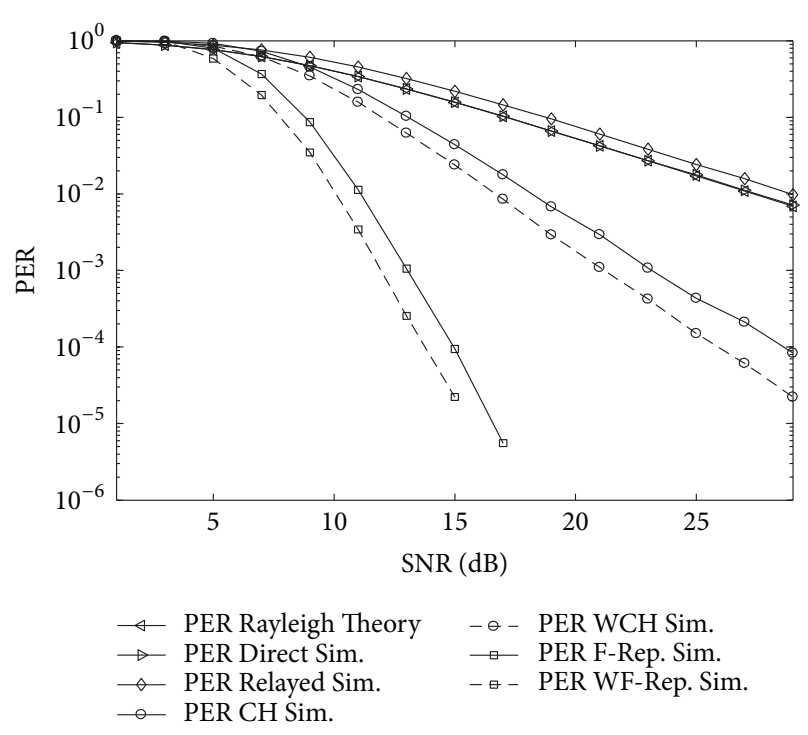

FIGURE 7: Comparison of the PER for direct, relayed, F-Rep. and $\mathrm{CH}$ cooperation showing the proposed weighted $\mathrm{CH}$ and weighted F-Rep. simulation results.

loss in performance due to using fewer nodes to relay the cooperative packet as compared to F-Rep. cooperation. Using the proposed RFFA mechanism, a further improvement of $\sim 2 \mathrm{~dB}$ and $\sim 1 \mathrm{~dB}$ is achieved in energy savings for $\mathrm{CH}$ and F-Rep. cooperation, respectively, at $10^{-3}$ BER. Thus, the $\mathrm{CH}$ cooperation scheme is able to reduce the latency and energy consumption of the network at the expense of some performance benefits. Using RFFA (WCH and WF-Rep. cooperation) allows us to save further energy and at the same time, improve the PER of the received data.

\section{Conclusion}

In this paper, we have proposed an algorithm called reliability factor feedback algorithm to improve the reliability of decisions made after sensor data fusion in relay-based cooperative WSNs to monitor the indoor industrial environment. We have analyzed the SER, power consumption, and latency of the proposed scheme. With the proposed algorithm, the reliability of the final decision has been increased significantly at the fusion center. Along with the increased reliability, significant energy savings have been achieved, which can be very beneficial in increasing the lifetime of the sensors. 


\section{Data Availability}

The code used to model the above described network and generate the provided results can be found at https://infonet .gist.ac.kr.

\section{Conflicts of Interest}

The authors declare that they have no conflicts of interest.

\section{Acknowledgments}

This work was supported in part by the National Research Foundation of Korea (NRF) Grant funded by the Korean government (MSIP) (NRF-2018R1A2A1A19018665) and in part by the Institute of Computing and Cybersystems (ICC) at Michigan Technological University.

\section{References}

[1] X. Bao and J. Li, "Adaptive Network Coded Cooperation (ANCC) for wireless relay networks: Matching code-on-graph with network-on-graph," IEEE Transactions on Wireless Communications, vol. 7, no. 2, pp. 574-583, 2008.

[2] J. N. Laneman and G. W. Wornell, "Distributed space-timecoded protocols for exploiting cooperative diversity in wireless networks," IEEE Transactions on Information Theory, vol. 49, no. 10, pp. 2415-2425, 2003.

[3] G. Kramer, M. Gastpar, and P. Gupta, "Cooperative strategies and capacity theorems for relay networks," IEEE Transactions on Information Theory, vol. 51, no. 9, pp. 3037-3063, 2005.

[4] A. Ulusoy, O. Gurbuz, and A. Onat, "Wireless model-based predictive networked control system over cooperative wireless network," IEEE Transactions on Industrial Informatics, vol. 7, no. 1, pp. 41-51, 2011.

[5] N. Marchenko, T. Andre, G. Brandner, W. Masood, and C. Bettstetter, "An experimental study of selective cooperative relaying in industrial wireless sensor networks," IEEE Transactions on Industrial Informatics, vol. 10, no. 3, pp. 1806-1816, 2014.

[6] J. Niu, L. Cheng, Y. Gu, L. Shu, and S. K. Das, "R3E: reliable reactive routing enhancement for wireless sensor networks," IEEE Transactions on Industrial Informatics, vol. 10, no. 1, pp. 784794, 2014.

[7] Y. M. Khattabi and M. M. Matalgah, "Performance analysis of multiple-relay AF cooperative systems over rayleigh timeselective fading channels with imperfect channel estimation," IEEE Transactions on Vehicular Technology, vol. 65, no. 1, pp. 427-434, 2016.

[8] J. Neuzil, O. Kreibich, and R. Smid, "A distributed fault detection system based on IWSN for machine condition monitoring," IEEE Transactions on Industrial Informatics, vol. 10, no. 2, pp. 1118-1123, 2014

[9] O. Kreibich, J. Neuzil, and R. Smid, "Quality-based multiplesensor fusion in an industrial wireless sensor network for MCM," IEEE Transactions on Industrial Electronics, vol. 61, no. 9, pp. 4903-4911, 2014.

[10] E. Fasolo, M. Rossi, J. Widmer, and M. Zorzi, "In-network aggregation techniques for wireless sensor networks: a survey," IEEE Wireless Communications Magazine, vol. 14, no. 2, pp. 7087, 2007.
[11] P. Jesus, C. Baquero, and P. S. Almeida, "A Survey of Distributed Data Aggregation Algorithms," IEEE Communications Surveys \& Tutorials, vol. 17, no. 1, pp. 381-404, 2015.

[12] H. Harb, A. Makhoul, R. Tawil, and A. Jaber, "Energy-efficient data aggregation and transfer in periodic sensor networks," IET Wireless Sensor Systems, vol. 4, no. 4, pp. 149-158, 2014.

[13] Z. Iqbal, K. Kim, and H.-N. Lee, "A cooperative wireless sensor network for indoor industrial monitoring," IEEE Transactions on Industrial Informatics, vol. 13, no. 2, pp. 482-491, 2017.

[14] Z. Iqbal and H. Lee, "Low-Latency and High-Reliability Cooperative WSN for Indoor Industrial Monitoring," in Proceedings of the 2017 IEEE 85th Vehicular Technology Conference (VTC Spring), pp. 1-6, Sydney, NSW, June 2017.

[15] R. Williams, The Geometrical Foundation of Natural Structure. A Source Book of Design, Dover, New York, NY, USA, 1979.

[16] F. Xue and P. R. Kumar, "The Number of Neighbors Needed for Connectivity of Wireless Networks," Wireless Networks, vol. 10, no. 2, pp. 169-181, 2004.

[17] Y. Ai, M. Cheffena, and Q. Li, "Radio frequency measurements and capacity analysis for industrial indoor environments," in Proceedings of the 9th European Conference on Antennas and Propagation (EuCAP 2015), p. 1, May 2015.

[18] A. F. Molisch, Wireless communications, John Wiley Sons, New York, NY, USA, 2011.

[19] W. H. Tranter, K. S. Shanmugan, T. S. Rappaport, and K. L. Kosbar, Principles of Communication Systems Simulation with Wireless Applications, Prentice Hall, Upper Saddle River, NJ, USA, 2004.

[20] IEEE 802.15.4-2011, Part 15.4: Low-rate wireless personal area networks (LR-WPANs), IEEE-SA Standards Board, 2011.

[21] "Silicon Labs, EFR32 Mighty Gecko Mesh Networking Wireless SoC," https://www.silabs.com/.

[22] K. Wu, C. Liang, K. Yu, and S. Kuang, "Multiple-mode floatingpoint multiply-add fused unit for trading accuracy with power consumption," in Proceedings of the 2013 IEEE/ACIS 12th International Conference on Computer and Information Science (ICIS), pp. 429-435, Niigata, Japan, June 2013. 


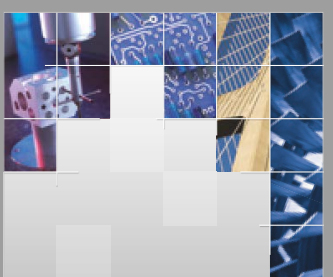

\section{Enfincering}
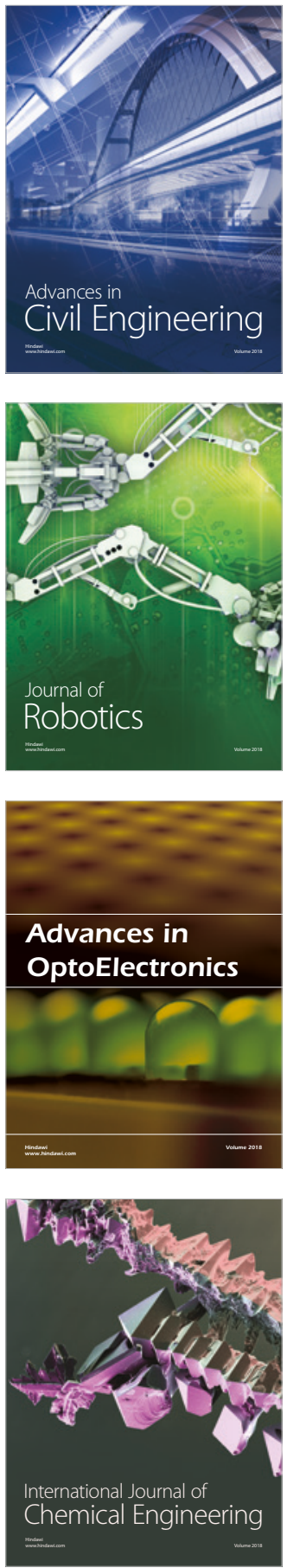

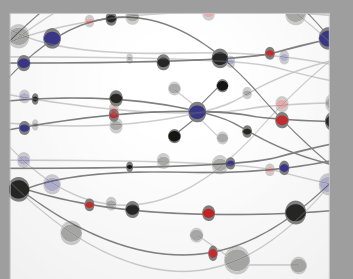

\section{Rotating \\ Machinery}

The Scientific World Journal

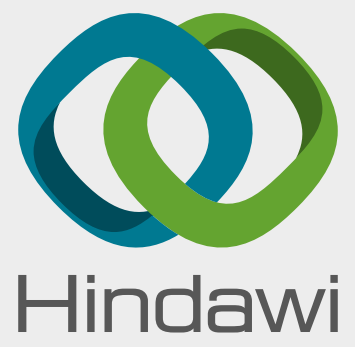

Submit your manuscripts at

www.hindawi.com
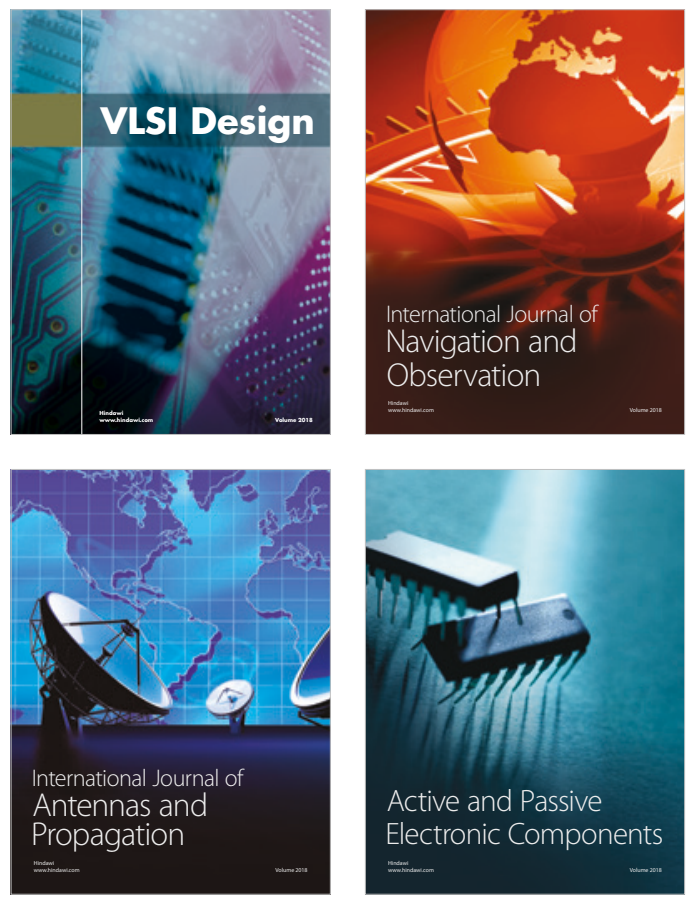
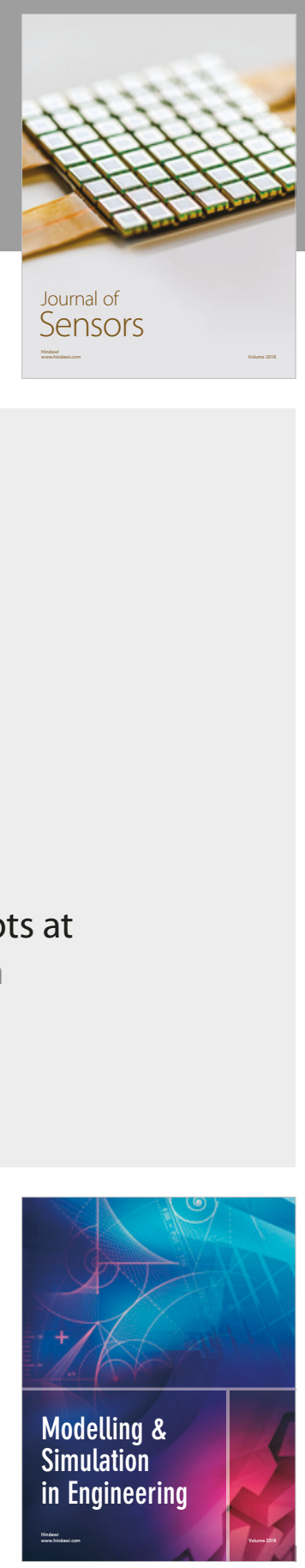

\section{Advances \\ Multimedia}
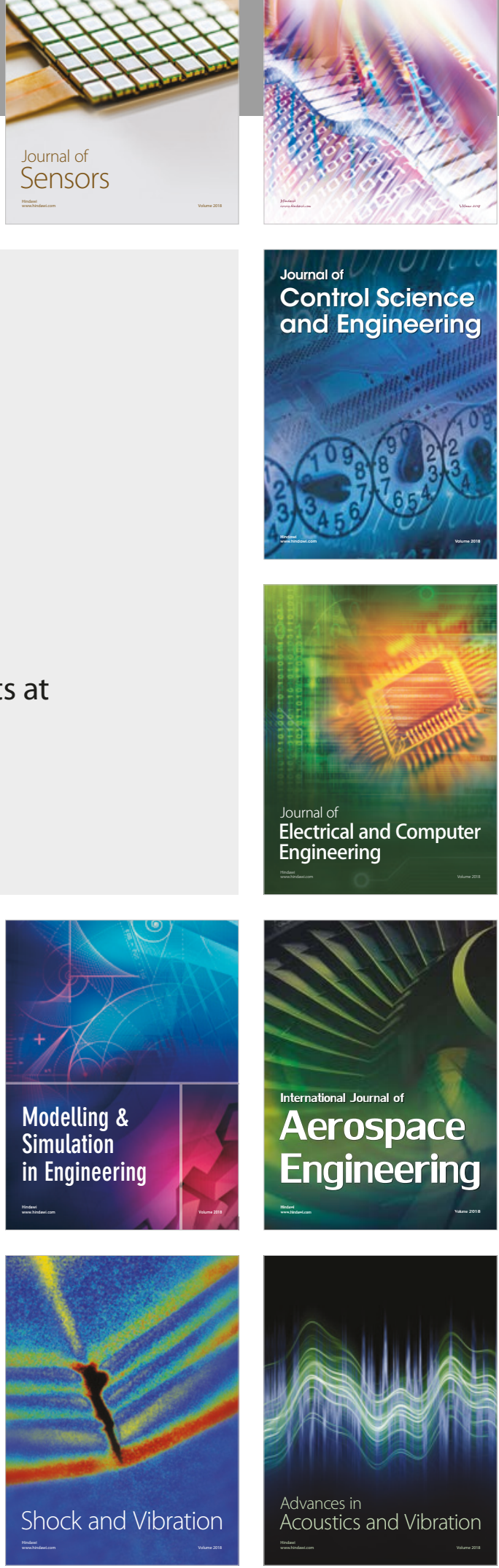